\title{
Téoros
}

Revue de recherche en tourisme

\section{Indicateurs du changement de la consommation touristique des Américains}

\section{Jean-Marc Bissonnette}

Volume 9, numéro 2, juillet 1990

Le marché américain

URI : https://id.erudit.org/iderudit/1080028ar

DOI : https://doi.org/10.7202/1080028ar

Aller au sommaire du numéro

Éditeur(s)

Université du Québec à Montréal

ISSN

0712-8657 (imprimé)

1923-2705 (numérique)

Découvrir la revue

Citer cet article

Bissonnette, J.-M. (1990). Indicateurs du changement de la consommation touristique des Américains. Téoros, 9(2), 19-22.

https://doi.org/10.7202/1080028ar d'utilisation que vous pouvez consulter en ligne.

https://apropos.erudit.org/fr/usagers/politique-dutilisation/ 


\section{Indicateurs du changement de la consommation touristique des Américains}

L'entreprise qui veut bien s'adapter aux conditions du marché américain a tout intérềt à bien connaître les habitudes de consommation de ses clients. Cela lui permet de mieux ajuster le bien ou service offert selon l'évolution du marche.

L'industrie touristique est particulièrement sensible aux grandes variations dans les habitudes de consommation. Àcet effet, on peut dégager un certain nombre de tendances chez nos voisins américains, pour la prochaine décennie, qui toucheront les modes de consommation touristique. II s'agit du vieillissement de la population, du repli sur soi (cocooning), de la rationalisation, de l'accroissement du temps de loisir, de la priorité accordée à la qualité du service et à la responsabilité corporative et de lat tendance qui veut que les riches seront de plus en plus riches.

Aucun instrument fiable n'existe en ce moment pour déterminer les grandes tendances de consommationdes Canadiens. Aux États-Unis, la firme Yankelovich, Clancy, Shulman ${ }^{(1)}$ fait chaque annee un sondage sur tout le territoire américain qui recueille les réponses de 2500 personnes. Cet instrument sert à identifier, mesurer et retracer les valeurs de base, les croyances et attitudes des consommateurs. Les données de cet indicateur peuvent être, de l'avis de plusieurs experts, extrapolées et appliquées au Canada mais, avec un certain décalage dans le temps. Le cadre de cet article tire son origine des données qualitatives issues de ce sondage et tentera $d^{*}$ appliquer ces elements au secteur touristique.

\section{Une population vieillissante}

Le vieillissement de la population n'est plus un secretpour personne. La génération des "baby boomer" dont on parle depuis si longtemps commence à prendre de l'âge. Phénomènes de l'après-guerre, le "baby boom" atteint son point culminant au milieu

\footnotetext{
Jean-Mare Bissonnette est agent de dávelopeement touristique à la CIDEM Nillo de Montróal).
}

des années ' 50 pour mourir au début des années '60. Onpeutétablirde façongénérale qu'en ce début des années "90, les "boomer" sont au mieux à la fin de la vingtaine ou dans la trentaine mais la plupart ont atteint maintenant le début de la quarantaine. D'ici quinzeans, ils atteindrontl'âge de la retraite.

Le vieillissement de la population n'est pas un phénomène exclusivement nordaméricain; au contraire, il est partagé par la plupart des pays industralisés. Examinons quelques statistiques à ce sujet: au milieu des années soixante, les 50 ans et plus comptaient pour environ $20 \%$ de la population, les 29 a 49 ans comptaient pour $31 \%$, et les "boomers", âgés de moins de 24 ans à cette époque, formaient presque $50 \%$ de la population. Vingt-cinq ans plus tard, ils ne forment plus que $38 \%$. Les projections pour $1^{*}$ an 2000 nous montrent a quel point le phénomène est loin de se résorber. En effet, on prédit que les proportions

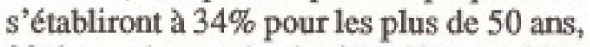
$38 \%$ pour la tranche des 25 aे 49 ans et $28 \%$ pour les moins de 24 ans. Cela constitue, si les prévisions se maintiennent, un déplacement de $14 \%$ entre la proportion des 50 ans et plus dans les années soixante et le toumant du $21^{\circ}$ siecle.

Ces données sont claires et il ne fait nul doute que les gens âgés de plus de cinquante ans, à l'aube du nouveau siècle, auront une très grande influence sur les comportements de consommation généraux et sur le type d'approche marketing qui devra être utilisé. Dejà quelques exemples probants ont commencé à semanifester. McDonald's utilise dans ses messages publicitaires des personnes âgles pour mousser la vente de ses produits auprès de cette nouvelle clientèle. Les banques font de même, en offrant des services spéciaux et rabais de toutes sortes aux aines. Les agences devoyages et les compagnies aériennes lorgnent du côté des gens du troisième âge et leur accordent une attention toute spéciale en vantant les mérites de leurs produits. Ce ne sont là que quelques-uns des exemples d'un phénomène qui ne cesse de prendre de $1^{\prime}$ ampleur. Mẹme si cela peut paraître encore troublant aujourd 'hui, cette clientèle sera de plus en plus sollicitée et, dans les années à venir, leurs besoins constitueront la norme. La majorité des individus qui composent cette nouvelle génération est peu endettée, les enfants ont grandi, quitte le foyernourricier, laissant papa et maman à la maison avec suffisamment d'argent pour être à l'aise et avec en poche du temps pour le dépenser.

Cette nouvelle répartition des groupes d'âge aura sans nul doute un effet très significatif sur l'industrie touristique. C'est une toute nouvelle clientèle qu'il faudra apprivoiser. Dès maintenant, les entreprises doivent tenir compte de cette nouvelle réalité dans la conception et la mise en marché de leurs produits.

\section{Le repli sur soi (cocooning)}

Les Américains ont donné à cette tendance le qualificatif de "cocooning". Celle-ci trouve son origine dans le fait que les personnes passent plus de temps a la maison, à l'abri d'une société de plus en plus complexe et oppressante. Ces individus recherchent d'abord un meilleur controble de l'aménagement de leur temps.

Ceux qui sont plus spécialement touchés par ces effets avaient l'habitude de manger àl'extérieur de la maison de cinq àsix soirs par semaine. Ils consacraient leur Energie entre le centre de sante et les bars et utilisaient leur temps libre à prendre tous les cours du soir qui leur tombaient sous la main. La maison demeurait simplement le lieu où l'on changeait de vêtements.

Ces mêmes individus, pour la plupart issus du "baby boom", tendent aujourd'hui à relấcher l'accélérateur et à accorder une place prépondérante àla vie au foyer. Rester à la maison n'est plus un mal en soi mais plutôt un bienfait.

Le confort à la maison devient leur première préoccupation au même titre que tout le côté sécurisant de leur foyer. Cette recherche d'intimité proviendrait du fait que la société, de l'avis de plusieurs, en demande trop. Le haut niveau de pression exigé au travail et l'anxiété reliée à la réussite d'une carrière font qu'il faut ralentir le 
rythme apres le boulot. II y a un grand besoin pour un autre type de vie, beaucoup plus privée et moins oppressante. $\hat{A}$ ce sujet, M. John Kettle, un consultant en marketing de la région de Toronto explique ce phenomène par le fait que les personnes qui avaient grandi dans la cohue des foules désiraient tout simplement être plus solitaires. Il n'est donc pas surprenant que la maison soit devenue un lieu de paix et de tranquillité et, par surcroît, un lieu où l'on resserre les mesures de contrôle sur son environnement.

Tous ces phénomènes liés à la vie dans les grandes villes que sont le courant de dépersonnalisation, la circulation difficile dans les centres-villes, $1^{4}$ augmentation de la criminalite, les foules, la fatigue et le coút de la vie éleve, sont autant de facteurs qui expliquentlatendance grandissante au repli sur soi (cocooning).

Ces pressions extérieures demandent un surcroit d'énergie que certains préféreraient dépenser à leur mieux être. $C^{y}$ est pourquoi, la plupart de ces individus consacrent le plus clair de leur argent $\mathrm{a}$ faire de leur foyer un lieu de refuge qui rencontre leurs besoins de confort et de securite.

L'effet du repli sur soi peut être facilement identifiéparuncertain nombre d'indicateurs présents sur le marché des biens de consommation. M. David R. Thexton du Creative Research Group de Toronto ${ }^{(2)}$, faisait remarquer, lors de sa presentation à la conférence du Tourism Outlook '90, qu'on observait depuis quelques années une augmentation dans les ventes des "divertissements maison". En effet, les ventes de magnétoscopes, d'un deuxième téléviseur, des disques au laser ont gonflé très rapidement. Les "commandes pour emporter ${ }^{\text {ti }}$ de son restaurant préféré ont progressé de façon importante. Larénovationdomiciliaire, l'ajout d'une piscine, les agrandissements de certaines pièces de la maison, l'amenagement d'une salle d'exercices, d'un spa et d'un jardin ont aussi connu des haussesentemes de ventes. Certains outils tels la telévision interactive, le téléphone cellulaire, 1 'interbranchement des ordinateurs personnels par modem et le télécopieur à la maison sont des produits des années ' 90 reflétant la tendance à quitter le bureau plus tôt pour travailler à la maison.

Le repli sur soi se traduit aussi dans le type de commodités et de confort recherché lorsque l'on va en vacances ou en weekend. L'hébergement du type auberge de campagne est de plus en plus populaire. Le nombre restreint de chambres, le charme de 1'endroit, le foyer, la bonne bouffe sont des critères hautement recherchés lors de la sélection d'un endroit pour ses vacances. "Se sentir a la maison loin de la maison", suffisamment loin de son lieu de résidence pour connaître le dépaysement mais dans un endroit où l'on peut retrouver le confort de son chez soi. L'impersonnalité des chambres des grands hôtels, la masse de gens qu'il faut cótoyer en ces lieux, dans le cadre des déplacements liés au travail, font que l'on recherche un tout autre type d'hébergement lorsqu'il s'agit de déplacements personnels.

\section{La rationalisation}

Selon Mme Marylin E. Sandler, présidente de The Creative Research Group Limited (3), de Toronto, qui a effectué des recherches sur la tendance a la rationalisation, cette nouvelle attitude concerne deux variables qui s'opposent dans la vie moderne, soit le "temps" et la complexité.

Qu'est-il possible de faire en tenant compte des ressources que nous possédons et comment fait-on les bons choix?

Durant les années '50, les choses étaient simples. On savait à cette époque ce qu'il fallait faire, et comment y arriver. $\hat{A}$ force de travail tout pouvait se réaliser. Faire son éducation, grandir, se marier, avoir des enfants, acheter une maison, une auto, prendre des vacances et finalement se retirer constituaient l'essentiel d'une vie satisfaisante et bien remplie. Le lavage se faisait le lundi, le repassage le mardi, l'épicerie le mercredi et ainsi de suite. S'il n'y avait pas assez d'argent ou de temps pour faire autre chose, cela faisait partie des contraintes de la vie.

Le consommateur des années 70 , selon l'étude faite par Mme Sandler, avait déjà bien évolué. Celui-ci croyait que tout était possible et qu'il s'agissait d'essayer de tout avoir. L'expérimentation était la règle et il y avait un désir constant de nouveautés. De nouveaux produits abondaient sur le marché durant cette décennie.

La nouvelle approche des années '90 tient beaucoup plus à un processus de rationalisation. Celui-ci met en évidence la théorie voulant que l'on peut faire ce que l'on désire vraiment faire mais qu'il n'est pas possible de tout faire.

Aujourd'hui et de plus en plus dans les années à venir, la femme devra concilier travail et maison, ce qui aura pour effet de la tenir passablement occupée. De plus, le nombre de familles monoparentales en augmentant constamment entraînera un stress additionnel pour le parent qui se retrouve seul avec des enfants. Ce demier n'a plus personne pour partager les tâches de la maison et les responsabilités se rattachant a la famille. On doit donc absolumentétablir des priorités et laisser de cốté tout ce qui n'est pas essentiel. Cela veut aussi dire de $1^{\prime}$ aide extérieure afin d'arriver à faire les choses jugées importantes et repousser celles qui gênent.

Cette tendance à la rationalisation affecte et affectera dans les annees ' 90 le processus de consommation. Premièrement, il y a un besoin de simplifier le processus de décision. A ce titre, le consommateur préférera tout ce qui lui est familier: marques connues, compagnies et produits fiables. Deplus, on choisira des experts à qui on déléguera notre responsabilité de faire le bon choix. Dans ce nouvel environnement, l'avantage sera donné à celui qui a la reconnaissance publique.

$\mathrm{L}^{*}$ aspect pratique des produits de consommation supportera fortement le mécanisme de rationalisation. L'accès facile al'information et aux services ainsi qu'aux endroits où l'on doit faire ses achats. Les produits devront aussi être facilement consommables: les diners congeles, la nourriture que l'on peut faire cuire au microondes, les contenants pratiques, les portions simples, etc.. Tout ceci et tout ce que l'on peut imaginer d'autres afin de rendre le produit plus simple et plus rapide à consommer. Le consommateur veut diminuer leplus possible le temps qu'il doit consacrer à l'achat et à la preparation des produits afin de s'en garder pour l'aspect le plus satisfaisant: la consommation ellemệme.

En ce qui a trait aux services touristiques, cette tendance sera unelément critique pour les années à venir. Prenons l'exemple du domaine des voyages. Lors dela préparation d'un voyage d'agrément, la qualité du service qui sera offert par l'agence de voyages sera bien souvent prédominante sur le produit lui-même.

Le consommateur ne tient plus à préparer son voyage seul a la maison en parcourant unequantité industrielle de guides. Il désire déléguer ce travail à son agent de voyages. Celui-ci devra préparer un programme complet du voyage, du départ de la maison en passant par les activités au lieu de destination, jusqu'au retour. Le produit Club Med se veut un bon exemple qui 
illustre bien cette tendance. Même s'il est peu abordable, il répond à un besoin, comme disent les Américains de "hassle free" (sans problème). Vous n'avez qu'à penser au bikini et à la brosse à dent, tout le reste est dejà fait pour vous. Pour cela, il faut que l'acheteur ait pleine confiance en lapersonne à qui il delègue sa responsabilité de choisir. Les forfaits tout compris sont les produits de l'avenir car ils n'ont qu'à se laisser consommer.

\section{L'accroissement du temps de loisir}

D'une façon génerale, le temps consacré aux activités de loisirs continuera d'augmenter au cours des années ' 90 avec la réduction du temps de travail. On assiste depuis un certain nombre d'annes à ce changement d'une société centrée essentiellement sur la valorisation du travail vers une société de loisirs. Cet accroissement du temps de loisirs, couplé a la valorisation des voyages, de l'Evasion, de l'exotisme, permettra au tourisme de connaître une croissance sensible.

Quelles seront les activités de loisirs en croissance pour la décennie a venir et comment se répartira le temps de vacances dans l'année?

Les ćmotions fortes et la fantaisie sont en vogue. Le temps de loisirs doit être occupé à briser la routine. Lamode est aux vacances où l'aventure est au premier plan: les safaris, la descente de rapides, le trekking dans les Himalayas, les voyages en Amazonie et plusieurs autres experiences du même type sont très en demande. L'accent est mis sur le risque contrôle, les Emotions fortes et l'aventure mais de façon prudente et sécuritaire.

Le concept de vacances change tranquillement, la faveur ctant aux longs week-ends aux Etats-Unis. En effet, on dénote un déplacement des traditionnelles vacances annuelles vers une sćrie de pauses de week-end. Ce morcellement implique une réduction du temps accordé aux longs congés annuels pour se garder une série de journees que 1'on cumule pour certaines fins de semaines choisies. A ce titre, un lien est à faire entre ce morcellement des vacances et la tendance à privilegier les établissements du type auberge, celui-ci s'adaptant mieux aux longs week-ends.

Le golf, le croquet et le billard ont connu une forte augmentation durant les dernières annés. Cette propension s'explique par le vieillissement de la population mais aussi par le fait que les individus issus de la wague du groupe des yuppies s'y intéressent de plus en plus.

L'augmentationdes ventes d'embarcations à moteur, du type yatch, au dépens des voiliers, constitue un autre indicateur du changement qui s'opère présentement dans le secteur des loisirs. Avec la fin de la crise de l'énergie, même en tenant compte des considerations ecologiques $\mathrm{d}^{5}$ aujourd hui, ce type de loisir a connu un essor assez considérable durant les demiéres annés et est appelé à continuer dans la mêne voie au cours de la prochaine décennie.

\section{La priorité à la qualité du service et à la responsabilité corporative}

L'industrie des services sera touchee dans les dix prochaines années par la tendance à prioriser la qualité du service et la responsabilité corporative avant toute autre chose. Ce nouvel enlignement des priorités change en quelque sorte les regles du jeu qui prevalaient dans le domaine du tourisme.

Voyons le contexte dans lequel cette tendance s'inscrit. Les voyages ne sont pas une nécessité mais plutôt un luxe. De ce fait, lorsque les revenus per capita montent ou descendent de un pourcent, les dépenses devraient suivre le même courant. Durant les années ' 50 et " 60 , le produit mondial brut augmentait de $6 \%$ par annee; dans les années 70 , il augmentait de $3 \%$ par annee; durant les années ' 80 par près de $2 \%$; et dans les annees '90, en raison de $1^{\prime} 6$ nome déficit américain et de celui du Royaume Uni, une diminution supplémentaire est prévue. L'impact de cette diminution devrait normalement se faire sentir dans le domaine des voyages. Il n'en est rien. En jetant un regard du côté des années ' 50 , on s'apercoit qu'à cette époque, seulement 25 millions de personnes par anné voyageaient en dehors de leur pays. $\mathbf{A}$ mesure que l'augmentation du produit mondial brut diminuait, le nombre de personnes qui voyageaient a l'extérieur de leur pays augmentait. Il se situe maintenant autour de 325 millions, soit une augmentation de quelque $1300 \%$ (4).

Il est clair que la libéralisationdeséchanges avec les pays de 1'Est va créer de nouveaux. marchés en Amérique du Nord. Par contre, la démocratisation de ces pays engendrera aussi de nouveaux compétiteurs sur nos propres marches et plus particulierement sur celui des États-Unis. On ne peut en ce moment $e$ valuer quels seront les impacts de cette ouverture mais sans nul doute plusieurs voyageurs seront tentés par ces nouveaux horizons. Certaines stratégiesdevront donc etre mises en oeuvre pour contrer cespertes. Dans la même veine, le processus de rapprochement de plusieurs pays pour former l'Europe de 1992 fera $d^{7} u n e$ multitude d'économies le plus grand marché au monde. La compétition qui s'engage peut se comparer en quelque sorte aux Olympiques. Les entreprises se battront contrelesmeilleures au monde, peu importe oùlles sont localisées. D'après le président du groupe "Four Seasons Hotels \& Resorts", M. Isadore Sharp, les vainqueurs seront ceux qui ont pris la resolution, de facon tress claire, de faire de la satisfaction du consommateurlapréoccupation no 1 . Cette theorie, mise de l'avant lors de la conférence "Tourism Outlook 90", soutient qu'il y a deux voies possibles pour les entreprises qui veulent atteindre le sommet, mais elles. se graviront sans plateau. Selon M. Sharp. "il faut faire place àl'innovation et améliorer sans cesse la qualite du service. Si nous pensons avoir atteint le point ou nous pouvons nous arrêter et reprendre notre souffle, c'est à ce moment que quelqu'un d'autre va prendre le leadership et se hisser devant nous".

Par ailleurs, le consommateur s'attend à oe que les entreprises soient responsables et prêtes a faire face a leurs obligations. Dans les années ' 50 , le consommateur s'accommodait de ce que les entreprises avaient à vendre. Le client achetait ce que le commerce mettait à sa disposition. Dans les années '70 cependant, le courant a completement changé de direction. Les besoins individuels sont devenus prioritaires avant ceux des entreprises, des gouvernements, de la religion, et de la famille. L'individu se sentait en droit d'exiger que les autres soient suffisamment flexibles pour répondre à ses besoins. Durant ces années, si quelque chose n'allait pas entre le consommateur et le vendeur, a coup sû́ la faute revenait au vendeur.

Aujourd'hui, les deux acteurs principaux, le consommateur et le commerçant, ont d'egales responsabilités. Le premier reconnait sa propre implication dans le processus $d^{7}$ achat et son obligation d'acquérir les qualités nécessaires pour prendre des décisions gagnantes. Le deuxième, quant à lui, devra répondre aux attentes des consommateurs en ce qui a trait a la compétence. Ceux-ci seront sans pitié 
pour les produits qui ne le satisferont pas pleinement. Si l'entreprise ne réussit pas à remplir les attentes de base de sa clientèle, celle-ci l'abandonnera tout simplement. L'environnement des années '90 ne permettra pas une seconde chance, ni les erreurs de parcours.

Avec la mondialisation des marchés, Montréal et Toronto seront toujours des destinations concurrentes, mais la vraie bataille se jouera peut-être à un tout autre niveau, celui de la planete. La place laissée aux petites entreprises sera de plus en plus restreinte. Celles-ci se feront avalerpardes "grands noms", Les multinationales peuvent, avec leur pouvoir d'achat, être partout à la fois. Le système de franchises prend d'ailleurs déjà de plus en plus d'ampleur. L'an 2000 nous apportera donc un moins grand nombre d'entreprises, les plus importantes se partageant souvent les mêmes marchés.

\section{Les riches seront de plus en plus riches}

L'écart entre les riches et les pauvres s'élargira durant la prochaine décennie, ce que révèlent plusieurs études de tendances. Cependant, onentrevoit qu'unmoins grand nombre de personnes seront riches a l'approche du XXIème siècle mais qu'elles seront plus riches proportionnellement à aujourd'hui. À l'inverse, plus de gens seront pauvres et de moins en moins de personnes feront partie de la classe moyenne.

Cette tendance n'est pas rose pour ceux qui tentent désespérément de rapprocher les deux extrêmes. Le message est pourtant très clair dans les communications qui émanent des firmes de consultants en marketing: les biens de consommation de luxe seront plus en vogue que jamais dans les dix prochaines années. Les individus n'auront plus aucun remord à posséder des objets cherset à vivre unstyle de vie luxueux.

Le consommateur qui aura les moyens de voyager sera prêt à y mettre le prix pour obtenir ce qu'il veut. Les forfaits de luxe seront nettement en demande croissante.

\section{Conclusion}

On peut dégager un portrait général de ce quepourraitêtre le consommateuraméricain de la prochaine décennie en regard de l'industrie touristique. On pourra reconnaittre deux grands groupesd'individus qui se subdiviseront en fonction de la variable âge.

Le premier, sans doute de plus en plus nombreux dans les années à venir, est issu du phénomène de la dénatalité et sera constitué de personnes qui seront ou bien à la retraite ou tout près de l'être. Assez bien nanties, elles auront du temps pour dépenser cet argent. Elles consommeront beaucoup de produits voyages et préféreront les forfaits tout compris. La qualité du service et la compétence corporative seront primordiales pour ce groupe de consommateurs.

Le deuxième groupe se situe dans le catégorie des $20-50$ ans et constitue l'essentiel de la force de travail. Les individus de ce groupe attacheront une importance toute particulière au bien-être au foyer. Ils sauront qu'ils peuvent faire ce qu'ils désirent mais qu'ils ne pourront pas tout faire. Grands consommateurs de produits pratiques et facilement consommables, ils préféreront au même titre que le premier groupe laisser àd'autres le soin de prendre les décisions pour eux. Ils prioriseront les entreprises qui seront en mesure de les assurer d'une qualité de service constante et qui rempliront leurs obligations.

Finalement, le consommateur riche dans les dix prochaines années sera, par comparaison avec aujourd'hui, encore plus riche. Il aura une propension très marquée vers les produits luxueux.

La prochaine décennie sera donc, pour le marché touristique américain, une décennie de grands changements en regard des habitudes de consommation. De ce fait, les efforts consacrés pour développer ce marché devront s'effectuer en constant arrimage avec l'évolution de la clientèle afin de maximiser les retombées de chacune des interventions. $f$

\section{NOTES}

[1) YANKELOVICH, CLANCY, SHULMAN, Yankelowich Monitor, Toronto, 1969.

(2) THEXTON, David R., The Creative Research Group Limited, rósumé de conférence. Toronto, novembre 1989.

(3) SANDLER, Marylin E., The Creative Research Group Limited, résumé de conférence, Toronto, avril 1989.

(4) SHARP, lsadore, Four Seasons Hotels \& Resorts, resumé de confúrence, Toronto, novembre 1969. 